J. Lake Sci. (湖泊科学), 2014, 26(4):497-504

http://www. jlakes. org. E-mail : jlakes@niglas.ac.cn

(c) 2014 by Journal of Lake Sciences

\title{
磷在“沉积物一自然生物膜-上覆水”三相体系中的迁移转化”
}

\author{
陆海鹰 $^{1}$, 陈建贞 ${ }^{1,2}$, 李运东 ${ }^{1}$, 方炎明 $^{2}$, 杨林章 ${ }^{1,3 * *}$, 吴永红 ${ }^{1 * *}$ \\ ( 1 : 中国科学院南京土壤研究所土壤与农业可持续发展国家重点实验室,南京 210008) \\ ( 2 : 南京林业大学森林资源与环境学院, 南京 210037) \\ ( 3 : 江苏省农业科学院农业资源与环境研究所,南京 210014)
}

\begin{abstract}
摘 要: 地表水体中沉积物表面通常会附着一层自然生物膜, 对上覆水一沉积物界面化学物质的迁移转化有着重要的影 响. 以往研究往往会忽略这一生物层面, 因而, 开展磷在 “沉积物一自然生物膜一上覆水”三相界面之间的迁移转化研究具 有重要的实际意义. 以软性填料表面形成的自然生物膜模拟沉积物表层以及悬浮颗粒物表层的自然生物膜, 开展磷在沉 积物一自然生物膜一上覆水之间的迁移转化过程研究. 结果表明: 有无曝气情况下, 自然生物膜界面的存在均能够显著降低 上覆水中总磷、溶解态总磷、溶解态无机磷的含量, 明显抑制沉积物中可交换态磷向上覆水释放. 研究证明自然生物膜在 水体磷的迁移转化中起着不可忽视的作用, 在研究沉积物一上覆水界面之间磷的迁移转化行为时, 要充分考虑自然生物
\end{abstract} 膜界面的存在.

关键词: 自然生物膜;磷; 曝气;沉积物; 上覆水

\section{Migration and transformation of phosphorus among the three-phase system "sediments- periphyton biofilm-overlying water"}

LU Haiying ${ }^{1}$, CHEN Jianzhen ${ }^{1,2}$, LI Yundong ${ }^{1}$, FANG Yanming ${ }^{2}$, YANG Linzhang ${ }^{1,3}$ \& WU Yonghong ${ }^{1}$

(1: State Key Laboratory of Soil and Sustainable Agriculture, Institute of Soil Science, Chinese Academy of Sciences, Nanjing 210008 , P. R. China)

(2: College of Forestry Resource and Environment, Nanjing Forestry University, Nanjing 210037, P. R. China)

(3: Institute of Agricultural Resources and Environment, Jiangsu Academy of Agricultural Sciences, Nanjing 210014, P. R. China)

Abstract: It is well known that periphyton biofilm is a kind of micro-aggregates that ubiquitously distributed in natural aquatic bodies, usually attaching on the sediment surfaces as well as the surfaces of suspended materials in waters. Therefore, it is more practical and meaningful to study the migration and transformation of phosphorus among “sediments-periphyton biofilm-overlaying water" system rather than the commonly sediments-overlaying water system. In this study, the periphyton biofilms used in experiments were collected from a kind of artificial substrate that designed special for the fast enrichment of periphyton, and then the migration and transformation of phosphorus among “sediments-periphyton biofilm-overlaying water" system was investigated. Results showed that the presence of periphyton biofilm interface could significantly decrease the content of total phosphorus, dissolved total phosphorus, dissolved inorganic phosphorus in overlying water under both situations of aeration and non-aeration, and obviously reduce the release of exchangeable phosphorus from sediments to overlying water. This study shows that it should take the periphyton biofilm interface into accounts when studying the phosphorus behaviors between sediments and overlying water.

Keywords : Periphyton biofilm; phosphorus; aeration; sediment; overlying water

随着污水治理技术、环保政策的日趋成熟以及湖泊污染治理力度的不断加大, 人湖污染物质等外源磷

* 国家自然科学基金项目 (41030640,41171363) 资助. 2013-07-24 收稿;2013-09-03 收修改稿. 陆海鹰（1986～), 男,博士研究生;E-mail:lhy2359595@126. com.

** 通信作者;E-mail: lzyang@ issas. ac.cn;yhwu@ issas. ac.cn. 
逐步得到控制甚至减少, 内源磷 $(\mathrm{P})$ 释放逐渐成为主要的污染因子 ${ }^{[1-2]}$, 沉积物中的磷吸附与释放使之逐渐 成为上覆水中磷的“汇”或者“源” ${ }^{[3]}$.

当前, 关于沉积物磷释放的研究主要集中在沉积物 (间隙水) 和上覆水 “两相”之间, 以磷的分级为出发 点研究磷在沉积物 (间隙水) 一上覆水之间的生物地球化学循环. 磷在沉积物 “相” 中一般分为交换态磷 (Exch-P)、铝结合态磷 (Al-P)、铁结合态磷 (Fe-P)、闭蓄态磷 (Bi-P)、原生碎屑磷 (De-P)、钙结合态磷

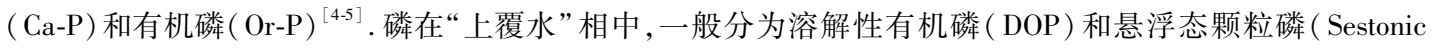
P), 分别占总磷的 $12 \% \sim 30 \%$ 和 $62 \% \sim 82 \%$, 生物可直接利用的溶解性反应磷约占 $5 \% \sim 8 \%{ }^{[6]}$. 沉积物磷 释放过程受到很多因素的影响, 如 $\mathrm{pH}^{[7]}$ 、温度 ${ }^{[8]}$ 、照度、溶解氧 $(\mathrm{DO})^{[9]}$ 、沉积物颗粒大小以及水生生物 ${ }^{[10]}$ 等, 尤其是水生生物状况对沉积物磷的地球化学行为有至关重要的影响, 生物过程对营养物质在水一沉积物 界面的迁移、源汇转换、形态变化与活化、钝化、固化方面起主要作用 ${ }^{[11]}$. 如沉积物磷的释放强度和频度因生 物种类、生物量、分布特点的改变而改变, 草型湖区、藻型湖区以及开阔湖面的沉积物的理化性质、间隙水中 的磷浓度及沉积物中磷的形态均存在较大差异 ${ }^{[12]}$. 沉水植物可以吸收富营养化湖水中的磷, 使上覆水中各 形态磷浓度均保持在较低的水平, 挺水植物的扰动也可以影响沉积物磷的释放过程 ${ }^{[13-14]}$. 因此, 研究磷在沉 积物一上覆水之间的迁移转化过程时,生物层因素值得深人探讨.

另外, 从污染控制角度而言, 长期以来我国对沉积物污染的控制一般采用两种措施: 一是采用底质封闭 的技术阻止底泥中污染物的释放; 二是采用底泥疏浚的方法彻底清除底泥 ${ }^{[15]}$. 由这个理念衍生的技术手段 还有诸如改变上覆水一沉积物界面环境, 通过原位覆盖、沉淀和针化等延缓内源磷释放 等 $^{[16]}$. 这些措施在一 定程度上有效地减缓了沉积物磷释放, 净化了上覆水水质, 但需要添加一些非湖泊自身生态组分的物质进 人湖体, 其对湖泊生态和地球化学循环的长远影响也有待进一步论证. 而底泥疏浚需要消耗大量的人力和 物力, 大规模应用会受到经济条件的限制. 因此, 依托水体生态系统自身的生态组分, 开发可以抑制沉积物 磷释放的绿色材料可能更具应用价值.

自然生物膜是指在自然环境条件下, 在淹水固体表面形成的微生物聚集体膜 ${ }^{[17]}$. 在自然水体中, 微生物 细胞和非生物物质镶嵌在微生物分泌的有机聚合物基质中, 并附着在固体表面 ${ }^{[18]}$. 微生物的胞外聚合物形 成了整个生物膜的骨架结构, 为微生物的附着生长和包裹吸附其它物质提供了物质基础. 从环境化学的角 度看,生物膜的定义普遍理解为由金属氧化物 (铁、锰和铝氧化物)、有机质和少量矿物质组成 ${ }^{[19-20]}$.

因此, 本研究引人地表水体中普遍存在但常被忽略的自然生物膜界面, 深人研究湖泊沉积物中磷的释 放形态和迁移特征, 即有利于我们深人了解沉积物磷的生物地球化学循环过程, 同时也为沉积物磷的释放 控制措施提供理论支持.

\section{1 材料与方法}

\section{1 自然生物膜富集与培养}

用玻璃缸培养自然生物膜, 主要过程为: 将工业弹性填料 (直径 $10 \mathrm{~cm}$, 长 $55 \mathrm{~cm}$ ) 放置在玻璃缸之后, 加 人从玄武湖采集的富营养水, 使富营养水长期淹没弹性填料, 每天置换一定的采集于玄武湖中的新鲜水. 培 养期间 $\mathrm{COD}_{\mathrm{Gr}}$ 为 $150 \sim 200 \mathrm{mg} / \mathrm{L}, \mathrm{pH}$ 为7.0 9. 0. 培养玻璃缸放置在户外, 培养条件如下: 水体温度在 $25^{\circ} \mathrm{C}$ 左右、曝气处理采用自动曝气装置 (曝气功率为 $12 \mathrm{~W}$ ), 自然光照. 经过 $15 \mathrm{~d}$ 的培养, 填料表面出现一层亮绿 色粘稠状物质, 此时认为已达到富集的目的, 然后用于下列实验.

\section{2 实验装置与实验设置}

玻璃实验装置长 $\times$ 宽 $\times$ 高 $=30 \mathrm{~cm} \times 20 \mathrm{~cm} \times 50 \mathrm{~cm}$, 装置底部装有湖泊底泥 $12 \mathrm{~cm}$, 上覆水 $24 \mathrm{~cm}$, 置于户 外. 底泥采自南京油坊桥附近一干涸不久的湖泊, 剔除其表层较干燥的土层, 采集深层湿润泥土, 经过篮除 大颗粒物质, 充分用水混合并搅拌均匀后倒人装置内. 上覆水为培养初期加人的模拟废水形成的高磷浓度 水(配方如下: $\mathrm{NaNO}_{3}: 0.2335 \mathrm{~g}, \mathrm{NH}_{4} \mathrm{Cl}: 0.0764 \mathrm{~g}, \mathrm{KH}_{2} \mathrm{PO}_{4}: 0.0150 \mathrm{~g}, \mathrm{MgSO}_{4} \cdot 7 \mathrm{H}_{2} \mathrm{O}: 0.0113 \mathrm{~g}, \mathrm{CaCl}_{2} \cdot 2 \mathrm{H}_{2} \mathrm{O}$ : $0.0014 \mathrm{~g}, \mathrm{FeCl}_{3} \cdot 6 \mathrm{H}_{2} \mathrm{O}: 0.0001 \mathrm{~g}, \mathrm{H}_{2} \mathrm{O}: 1000 \mathrm{~kg}$ ), 开始采样分析后不再加人营养水质, 由于蒸发等造成的损 失量由自来水补给, 使水位维持恒定. 实验装置分为 3 组: 第 1 组为只有底泥和上覆水的对照组; 第 2 组中除 底泥和上覆水外,包括富集和培养的自然生物膜; 第 3 组则在第 2 组的基础上另外增加增氧机表面曝气, 模 
拟湖泊自然状态下的扰动状态. 实验时间为 2012 年 5 月 26 日一 7 月 26 日,每隔 $15 \mathrm{~d}$ 取样 1 次进行相关指标 测试. 以上每组实验设置 3 个重复.

\section{3 实验分析方法}

上覆水总磷 (TP) 浓度测定采用过硫酸钾氧化法; 将水样预先过 $0.45 \mu \mathrm{m}$ 孔径的醋酸纤维滤膜,而后采 用过硫酸钾氧化法测定溶解性总磷 (DTP) 浓度; 而后采用锄一锑一抗比色法测定溶解性无机磷 (DIP) 浓 度 ${ }^{[21]}$; 颗粒磷 $(\mathrm{PP})$ 浓度为 TP 与 DTP 浓度之差; 溶解性有机磷 $(\mathrm{DOP})$ 为 DTP 与 DIP 浓度之差.

利用高氯酸-硫酸消化法进行消解后测定生物膜的含磷量 ${ }^{[22]}$. 采用自制简便装置从泥水界面采集表层 $0 \sim 3 \mathrm{~cm}$ 沉积物后, 经过风干, 过 100 目篮后测定沉积物含水量和其它项目. 沉积物中各形态磷含量参考文 献 [23-24]进行测定.

数据处理与分析利用 Excel 和 SPSS 19.0 软件进行, $t$ 检验用来比较对照组和处理组是否具有统计学 差异.

\section{2 结果与讨论}

\section{1 自然生物膜特征}

利用扫描电镜观察自然生物膜可以看 出,在实验中富集得到的自然生物膜主要由 硅藻和细菌组成 ( 图 1). 硅藻主要由以下几 种构成: 双头针杆藻 (Synedra ulna Kütz.)、 异极藻 (Gomphonema parvulum Kütz. )、翁格 脆杆藻 (Fragila riavaucheriae Kütz.) 、变异直 链藻 (Melosira varians Ag.) 和双头菱形藻 (Nitzschia amphibian Grun. ).

\section{2 自然生物膜生物量及其磷含量}

通过对实验中两个处理组的生物膜生 物量进行测量 (鲜重), 可以看出增加曝气的 生物膜生物量明显高于没有曝气的处理 (图 $2 \mathrm{~A})$, 且达到显著水平 $(P<0.05)$. 由图 $2 \mathrm{~B}$ 可知,无论曝气与否, 随时间增长, 单位生物 膜含磷量呈明显增大趋势, 且曝气与不曝气 相比无显著差异 $(P>0.05)$, 这可能是因为

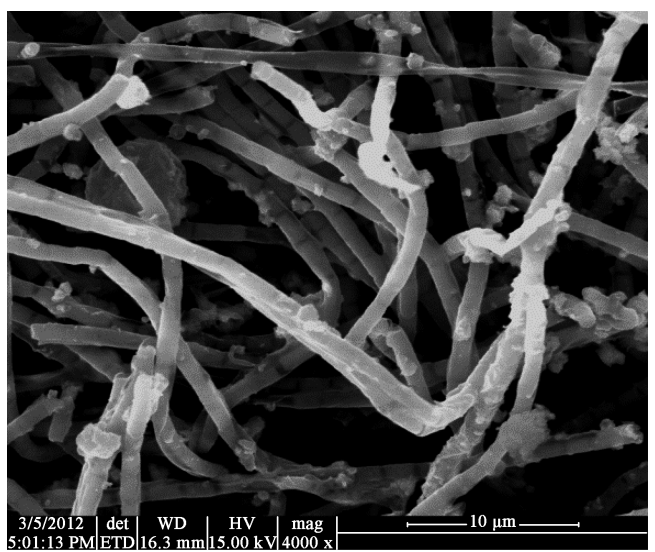

图 1 通过扫描电镜观测的自然生物膜图片

Fig. 1 Photo-image of periphyton biofilm characteristilized by scanning electron microscope 曝气导致生物膜量的增加,但没有增加其除磷能力. 在实验第 $15 \sim 30 \mathrm{~d}$ 时生物膜总含磷量增幅最大, 在 $45 \mathrm{~d}$ 后, 逐渐趋于平稳, $60 \mathrm{~d}$ 后, 生物膜总含磷量达到最大, 无曝气处理由 $72.13 \mathrm{mg}$ 增加到 $131.78 \mathrm{mg}$, 曝气处理 由 $69.03 \mathrm{mg}$ 增加到 $139.25 \mathrm{mg}$ (图 2C).

生物膜中磷含量可以反应出生物对磷的吸收能力, 从而可以认识到生物的生长对系统中磷的影响. 同 时, 由于生物膜的新陈代谢以及代谢物质的影响, 从而影响到沉积物中磷的变化. 初期外界条件适宜, 上覆 水中有丰富的营养盐, 生物膜生长迅速, $30 \mathrm{~d}$ 后随着营养物质的逐渐减少, 生物膜逐渐由生长成熟期转变为 稳定期以及衰退期,生物量趋于稳定. 曝气有利于自然生物膜生物量的累积, 这可能是由于充足的氧气导致 一些好氧型细菌和藻类快速生长. 此外, 曝气除了增加水体氧气含量之外, 也增加了水体的流动, 从而增加 了水体中营养盐与生物膜的交换. 但是,伴随着自然生物膜生物量的增加, 在整个实验期间,生物膜中总含 磷量始终在增加, 曝气对生物膜总含磷量的影响并未达到显著水平 (图 $2 \mathrm{C}, P>0.05$ ). 一方面可能是曝气导 致生物膜生物量的增加,但是抑制了对磷具有吸收作用的细菌或藻类的增加, 比如某些厌氧细菌. 另一方面 很可能是因为自然生物膜生物量的增加往往会形成一层致密层 ${ }^{[25]}$, 而这层致密层几乎对磷的吸收不起作 用,仅仅导致生物量的徒增. 

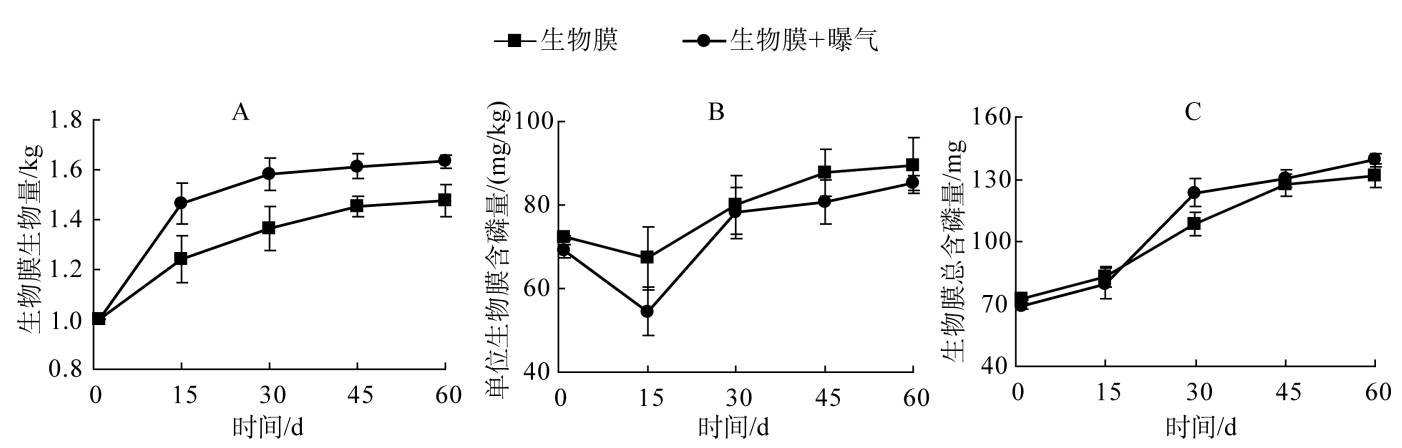

图 2 自然生物膜生物量及磷含量变化

Fig. 2 The changes of biomass and phosphorus content of the periphyton biofilm

\section{3 上覆水中各形态磷含量的变化}

水中磷含量变化直接和水体中藻类、细菌和真菌的生长密切相关. 当外界环境适宜, 且水体中氮、磷浓 度, 特别是限制因子磷浓度满足微生物的生长要求时, 湖泊水华暴发的风险明显增大. 水体中磷的形态大体 分为可溶性总磷、溶解性磷酸盐, 其中溶解性磷酸盐可以直接被水生生物利用.

实验过程中所取用的上覆水 TP、DTP 和 DIP 的初始浓度分别为 $0.99 \pm 0.02 、 0.89 \pm 0.018$ 和 $0.74 \pm$ $0.017 \mathrm{mg} / \mathrm{L}$. 对照组上覆水 TP、DTP、DIP 浓度随着时间的增加呈上升趋势, 而 PP、DOP 浓度在实验期间基本 平稳, 没有明显波动 (图 3), 表明沉积物底泥在实验期间存在一个明显的磷释放过程. 自然生物膜存在的实 验组上覆水 TP、DTP、DIP 浓度则成单峰趋势变化, 在实验进行到第 $15 \mathrm{~d}$ 时添加生物膜的装置中 (无论曝气 与否) TP、DTP 和 DIP 的浓度分别达到最大值, $15 \mathrm{~d}$ 后随着时间的推移而下降, $45 \mathrm{~d}$ 后趋于稳定; 与对照组相 比, 生物膜的存在显著降低了上覆水中 TP、DTP、DIP 的含量 $(P<0.05)$, 这表明自然生物膜的存在可以显著 降低上覆水中磷浓度, 从而减小水体富营养化发生的风险.

通过对上覆水各形态磷含量随时间的变化特征分析可以看出, “沉积物一生物膜一上覆水”三相系统中, 自然生物膜的存在使上覆水中 TP、DTP 和 DIP 浓度明显降低 (图 3). McCormick 等 ${ }^{[26]}$ 认为自然生物膜在水 体中常充当 “磷库” 的作用, 自然生物膜在沉积物一水界面的存在对磷的去除、吸收、转化以及生物有效性等 方面都起着非常重要的作用 ${ }^{[27-28]}$. 对比图 3D、图 3E 可以看出, 生物膜对 DIP 的影响要大于 DOP, 这是因为 水体中可被生物利用的磷多为 DIP, 随着生物膜的生长, 硅藻以及水体中其他藻类不断生长, 上覆水中大量 的无机磷被吸收. 另外, 增加曝气的处理组在 $15 \mathrm{~d}$ 时其 PP 达到最大值 $(0.30 \pm 0.0015 \mathrm{mg} / \mathrm{L})$, 这可能是由于 曝气人为增加了 “沉积物-生物膜一上覆水” 系统的扰动, 使沉积物表面的颗粒性磷随着扰动混人上覆水, 使 其含量增加; 随后便呈下降趋势直至趋于稳定, 这可能要归因于自然生物膜的吸附作用, 自然生物膜的胞外 聚合物与泥污生物膜很相似,具有更多可以吸持污染物的吸附位点 ${ }^{[29-30]}$.

\section{4 沉积物中各形态磷含量的变化}

沉积物中磷含量及其形态分布是影响湖泊富营养化进程极为重要的因素之一. 有研究认为, 沉积物磷 的释放可使水体的富营养化问题持续数十年 ${ }^{[31]}$, 因此, 控制沉积物磷的释放刻不容缓. 通过对比添加自然生 物膜的处理组与对照组沉积物中的 TP、Exch-P、NH $\mathrm{NH}_{4} \mathrm{Cl}-\mathrm{P}$ 随时间变化规律 (图 4), 不难发现, 无论处理组还是 对照组在实验初期沉积物 TP 总是处于下降状态, 随着实验时间的延长, 在后期沉积物 TP 逐步趋于稳定, 且 处理组与对照组 TP 含量变化表现出显著差异 $(P<0.05)$, 这表明沉积物在实验期间不断向水环境中释放 磷, 而生物膜的存在并未改变这种释放趋势, 但显著改变了沉积物磷释放强度. 同时观察发现, 在曝气 (人为 扰动) 的环境条件下, 沉积物中 TP 相比于无曝气处理, 下降幅度稍大, 这也意味着曝气处理可能使得 “沉积 物-生物膜-上覆水”系统扰动更为频繁,促进了系统中营养元素如磷的迁移交换.

Exch-P 主要是指沉积物中氧化物、氢氧化物以及黏土矿物颗粒表面等吸附的磷, 有研究表明沉积物中 的 Exch-P 是富营养化水体中蓝藻和“水华” 生长的主要磷源 ${ }^{[32]}$. 由图 4B 可以发现, 无论对照组还是实验处 理组, 沉积物中的 Exch-P 随着时间的增加都呈下降趋势, 添加自然生物膜的处理组在 $45 \mathrm{~d}$ 后逐渐趋于平 


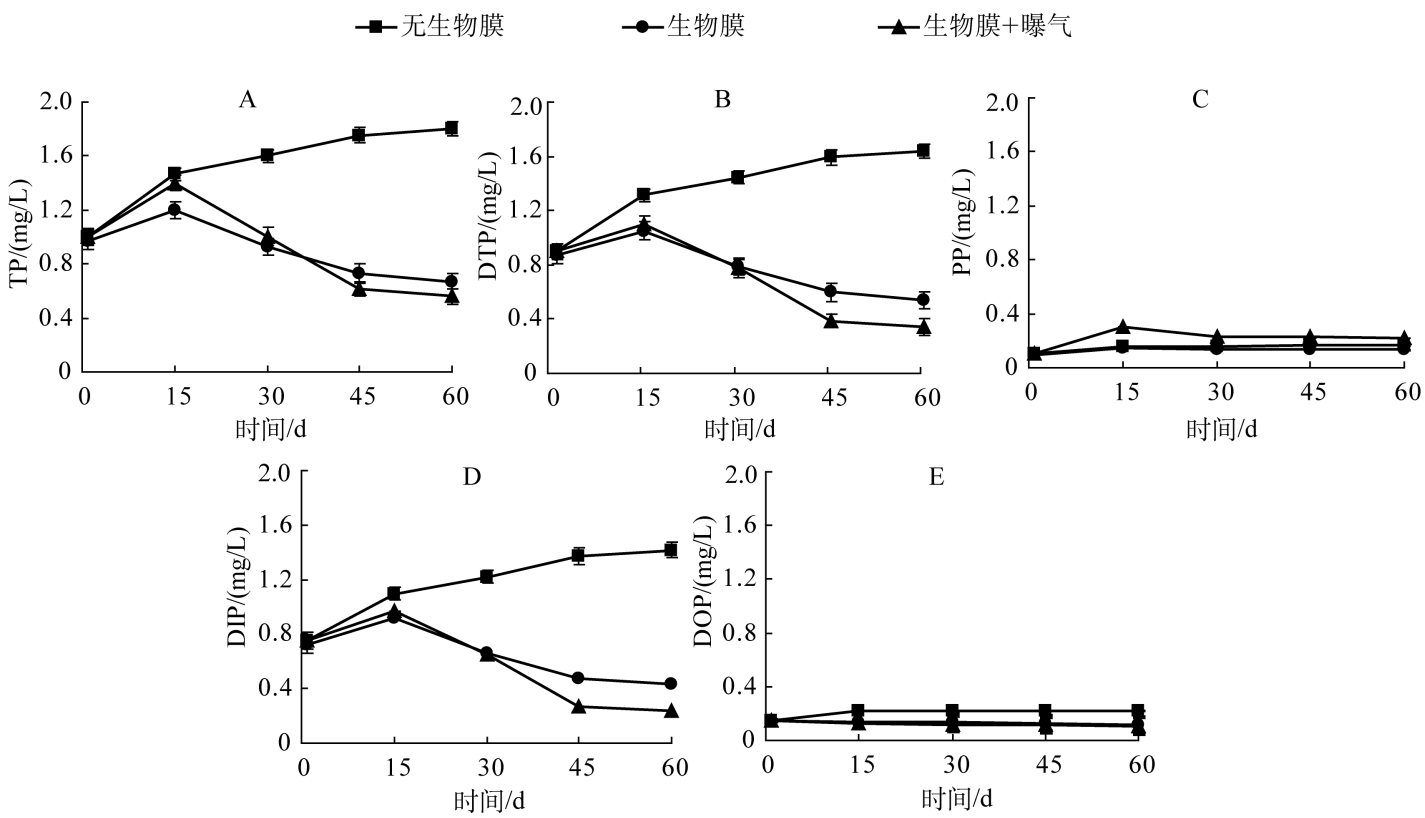

图 3 上覆水中 TP、DTP、PP、DIP 和 DOP 浓度的变化

Fig. 3 Changes of TP, DTP, PP, DIP and DOP concentrations in the overlying water

稳, 对照组由最初的 $6.32 \mathrm{mg} / \mathrm{kg}$ 下降到 $4.86 \mathrm{mg} / \mathrm{kg}$, 其下降程度与处理组相比达到显著水平 $(P<0.05)$, 说 明自然生物膜在沉积物一上覆水界面的存在显著减少了沉积物中 Exch-P 的释放. 在曝气处理下, 沉积物 Exch-P 也表现出相同的变化趋势,但与无曝气处理差异不显著 $(P>0.05)$. 本结果与前人研究结果一致,证实 了沉积物表面生物膜的存在不仅可以吸收水界面中的营养盐, 还可以通过光合呼吸作用等改变沉积物一水 界面氧化还原电位以及 $\mathrm{pH}$, 从而抑制沉积物界面磷释放 ${ }^{[33-34]}$.

弱吸附态磷 $\left(\mathrm{NH}_{4} \mathrm{Cl}-\mathrm{P}\right)$ 属于易释放态磷, 这类形态的磷极易被生物利用 ${ }^{[35]}$, 从图 4C 可以看出, 不论是 否添加自然生物膜,沉积物中的 $\mathrm{NH}_{4} \mathrm{Cl}-\mathrm{P}$ 总体变化趋势不变,都呈现初期下降,后期 $(30 \mathrm{~d}$ ) 逐渐趋于稳定的 趋势,而添加自然生物膜的处理组 $\mathrm{NH}_{4} \mathrm{Cl}-\mathrm{P}$ 下降程度较对照组轻, 分别由原来的 $0.38 \mathrm{mg} / \mathrm{L}$ 下降到 $0.14 \mathrm{mg} / \mathrm{L}$ (无曝气) 和 $0.15 \mathrm{mg} / \mathrm{L}$ (曝气), 表明自然生物膜的存在可以减少 $\mathrm{NH}_{4} \mathrm{Cl}-\mathrm{P}$ 的释放.

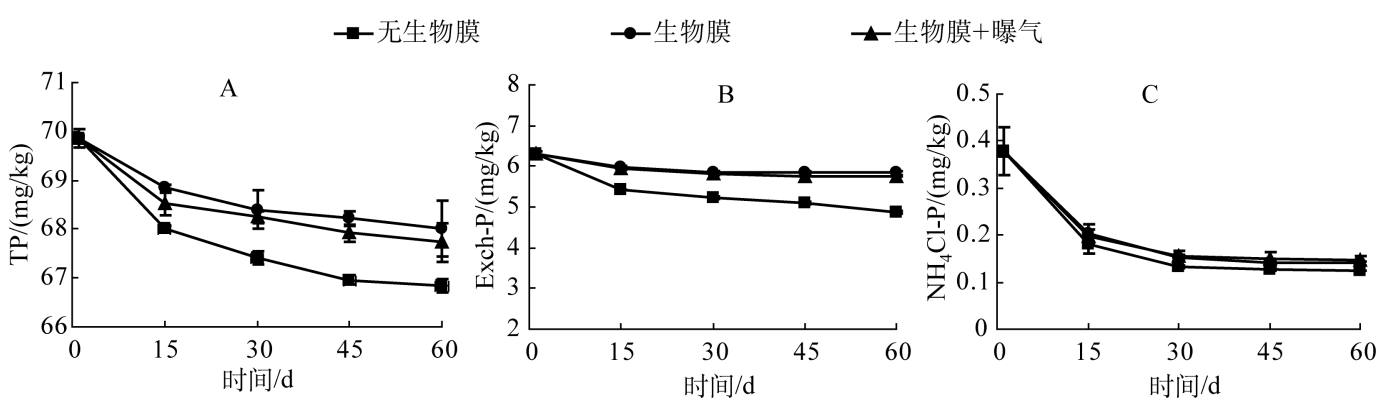

图 4 沉积物中 TP、Exch-P 和 $\mathrm{NH}_{4} \mathrm{Cl}-\mathrm{P}$ 浓度的变化

Fig. 4 Changes of TP, Exch-P and $\mathrm{NH}_{4} \mathrm{Cl}-\mathrm{P}$ concentrations in the sediment

为进一步了解沉积物中其他形态磷的迁移转化规律, 本实验还对沉积物的钙结合态磷 ( Ca-P)、铝结合 态磷 ( Al-P)、铁结合态磷 ( Fe-P) 和闭蓄态磷 ( Oc-P) 进行测定. Ca-P 在沉积物中属于相对惰性的一种磷, 这 是由于其自身较高的溶解度常数. 对照组 $\mathrm{Ca}-\mathrm{P}$ 的含量在实验过程中随时间延长基本保持不变,但有小幅波 
动 (图 5A). 不论曝气与否, 自然生物膜存在的处理组 Ca-P 在整个实验过程中都呈上升趋势, 在 $30 \mathrm{~d}$ 时达到 最大值, 之后便逐渐稳定, 且末期 Ca-P 含量与对照组差异显著 $(P<0.05)$, 这表明自然生物膜的存在增加了 沉积物中 Ca-P 的含量. Al-P 与 Fe-P 具有很强的释放活性, 又被称为活性磷, 是内源负荷的重要来源. 对照组 中沉积物的 Al-P 和 Fe-P 随时间延长都呈下降趋势 (图 5B、图 5C), 而添加自然生物膜的处理组 (无论曝气 与否) 与对照组中 Al-P 和 Fe-P 含量变化均达到显著差异 $(P<0.05)$, 这表明自然生物膜的存在有效遏制了 沉积物中 Al-P 和 Fe-P 向水体中迁移, 这也与上述可交换态磷的结果相一致. Oc-P 是沉积物中某些矿物颗粒 ( $\mathrm{Fe} 、 \mathrm{Al}$ 等) 包裹磷所形成的,一般较难释放 ${ }^{[34]}$. 对照组与处理组沉积物中 Oc-P 含量在实验过程中没有显著 变化,自始至终保持在某一稳定值 $(4.34 \pm 0.025 \mathrm{mg} / \mathrm{kg}$ ) ( 图 5D).

一般环境条件下, Ca-P 很难分解参与短时间的磷循环 ${ }^{[36]}$, 沉积物中的 Ca-P 主要包括两部分, 即与某些 自生碳酸钙共同沉淀的磷以及河流或者湖泊中风化侵蚀产物中的磷灰石岩等. 分析对照组与处理组 Ca-P 变 化特征可以发现, 自然生物膜的引人使上覆水相关理化性质改变, 藻一菌生物膜的投人可以改变水体的 $\mathrm{pH}$ 值 $^{[37]}$, 初期自然生物膜的生长旺盛, 水体 $\mathrm{pH}$ 值上升呈现碱性, 在这种环境条件下, 钻离子极易形成沉淀, 同 时与某些磷结合, 以钙沉淀一磷复合形式共同沉降, 导致这个时间 Ca-P 含量上升. 后期随着自然生物膜生长 成熟, 逐渐平稳, 沉积物中 Ca-P 含量逐渐趋于稳定.

有机质对 Al-P、Fe-P、Oc-P 这 3 种形态磷的含量在某种程度上影响较大 ${ }^{[38]}$. 许金树等 ${ }^{[39]}$ 指出 Al-P 和 Fe-P 可以作为指示沉积物质量的重要指标之一. 由于自然生物膜的存在, 使系统中有机质含量升高, 有机质 在降解过程中所释放的溶解态磷被 $\mathrm{Fe} 、 \mathrm{Al}$ 的 (氢) 氧化物吸附, 沉降, 所以在实验初期, 沉积物中 $\mathrm{Al}-\mathrm{P}$ 有小幅 升高, 但是随着沉积物中氧化还原环境的形成与稳定, Al-P、Fe-P 在沉积物氧化还原条件的影响下可以转化 成溶解态磷 (DP), 通过间隙水进人上覆水体, 进而被生物膜吸收, 所以 $15 \mathrm{~d}$ 后 Al-P、Fe-P 呈下降趋势. 有研 究指出, 在厌氧环境条件下, 沉积物中 Fe-P 能够释放以供环境中生物的生长 ${ }^{[40]}$. Oc-P 主要包括两类: 闭蓄态

- - 无生物膜
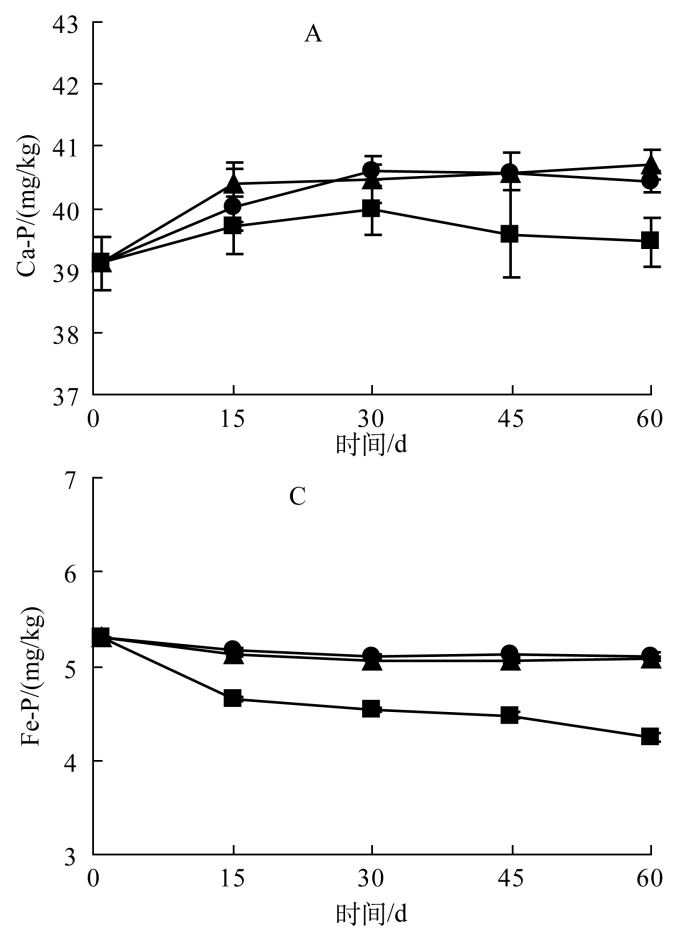

- - 生物膜
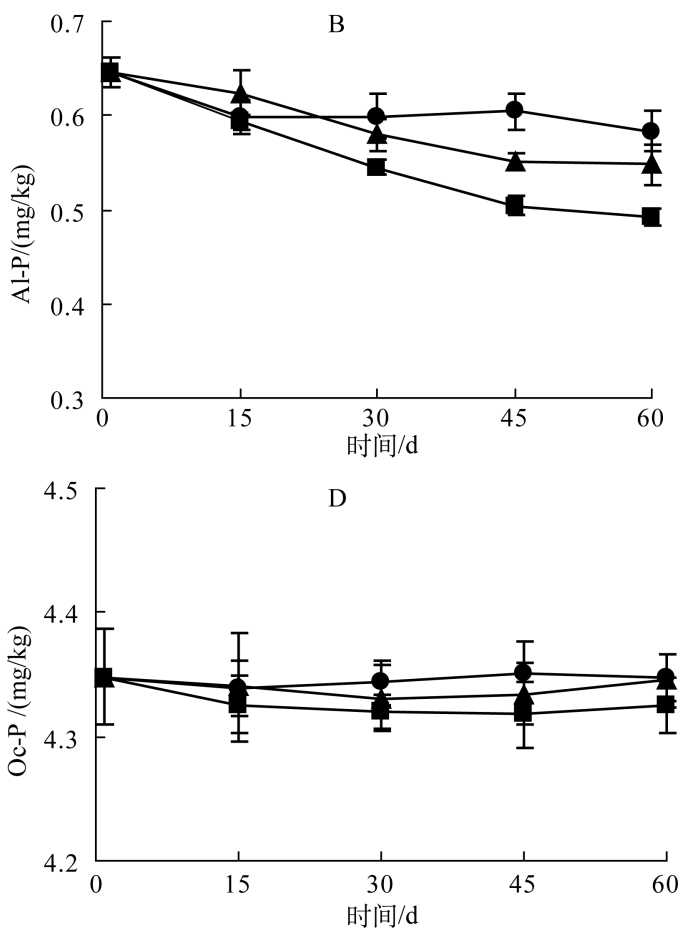

图 5 沉积物中 Ca-P、Al-P、Fe-P 和 Oc-P 浓度的变化

Fig. 5 Changes of Ca-P, Al-P, Fe-P and Oc-P concentrations in the sediment 
$\mathrm{Al}$ 磷 ( Oc-Al-P) 和闭蓄态 $\mathrm{Fe}$ 磷 ( $\mathrm{Oc}-\mathrm{Fe}-\mathrm{P})$, 其实质是被 $\mathrm{Fe}_{2} \mathrm{O}_{3}$ 胶膜所包裹的具有还原性的磷酸铁与磷酸铝, 在沉积物中与 $\mathrm{Fe}-\mathrm{P}$ 性质相似, 容易受到周围环境的 $\mathrm{Ph}$ 、氧化还原电位 ( $\mathrm{Eh}$ 和 $\mathrm{Es}$ ) 的影响. 但是, 本实验结果 发现 Oc-P 含量并没有显著性变化, 这可能是因为它在非还原条件下很难被其他生物吸收利用( 比如自然生 物膜),这部分磷在这个系统中就很难参与沉积物、水、生物膜的磷循环.

\section{3 总结}

1) 沉积物中的磷是水体富营养化的一个重要来源,在适宜的条件下可以通过间隙水向上覆水体释放, 影响着水体的富营养化程度. 本实验表明自然生物膜的存在能够有效降低沉积物中可交换态磷与弱吸附态 磷向上覆水的释放, 使沉积物一上覆水中磷的迁移发生显著变化, 因此研究沉积物一上覆水界面磷的迁移转 化过程必须要考虑到生物层的存在.

2) 水体的富营养化主要是由水体中氮、磷浓度过高造成的,通过观察对照组与处理组中上覆水以及沉 积物各形态磷含量变化可以发现, 自然生物膜的存在不仅可以降低水体中磷的含量, 并能在抑制沉积物中 磷的释放,因此基于自然生物膜的水体富营养化生态修复工程具有巨大的潜在应用前景.

由此可以看出,依托湖泊生态系统自身的生态组分一一自然生物膜,不仅能在一定程度上净化上覆水 水质,而且能有效地减缓沉积物磷释放, 从而缓解某些高磷水体的富营养化程度.

\section{4 参考文献}

[ 1 ] 范成新,张 路,王建军等. 湖泊底泥疏浚对内源释放影响的过程与机理. 科学通报,2004,49(15):1523-1528.

[ 2 ] Palmer-Felgate EJ, Bowes MJ, Stratford C et al. Phosphorus release from sedimentsin a treatment wetland: Contrast between DET and $\mathrm{EPC}_{0}$ methodologies. Ecological Engineering, $2011,37(6)$ : 826-832.

[ 3 ] Slomp CP, Malschaert JFP, van Raaphorst W. The role of adsorption in sediment-water exchange of phosphate in North Sea continental margin sediments. Limnology and Oceanography, 1998 , 43(5) : 832-846.

[ 4 ] 李 悦,乌大年,薛永先. 沉积物中不同形态磷提取方法的改进及其环境地球化学意义. 海洋环境科学, 1998,17 (1) : $15-20$.

[ 5 ] Ruttenberg KC. Development of a sequential extraction method for different forms of phosphorus in marine sediments. Limnology and Oceanography, 1992, 37(7) : 1460-1482.

[6] 高光,朱广伟,秦伯强等. 太湖水体中碱性磷酸酶的活性及磷的矿化速率. 中国科学: D 辑: 地球科学, 2005,35 (增刊) : 157-165.

[ 7 ] Jin X, Wang S, Pang Y et al. Phosphorus fractions and the effect of $\mathrm{pH}$ on the phosphorus release of the sediments from different trophic areas in Taihu Lake, China. Environmental Pollution, 2006, 139 : 288-295.

[ 8 ] Liikanen A, Murtoniemi T, Tanskanen $\mathrm{H}$ et al. Effects of temperature and oxygenavailability on greenhouse gas and nutrient dynamics in sediment of a eutrophic mid-boreal lake. Biogeochemistry, 2002,59 : 269-286.

[ 9 ] Jin X, Jiang X, Yao Y et al. Effects of light and oxygen on the uptake and distribution of phosphorus at the sediment-water interface. Science of the Total Environment, 2006, 357 (1/2/3): 231-236.

[10] Gainswin BE, House WA, Leadbeater BSC et al. Kinetics of phosphorus release from a natural mixed grain-size sediment with associated algal biofilms. Science of the Total Environment, 2006, 360(1/2/3) : 127-141.

[11] Palmer-Felgate EJ, Mortimer RJG, Krom MD et al. Internal loading of phosphorus in a sedimentation pond of a treatment wetland: Effect of a phytoplankton crash. Science of the Total Environment, $2011, \mathbf{4 0 9}(11): 2222-2232$.

[12] 朱广伟,高 光,秦伯强等. 浅水湖泊沉积物中磷的地球化学特征. 水科学进展,2003,14(6):714-719.

[13] Jukka H, Leena N. Effects of submerged macrophytes on sediment resuspension and internal phosphorus loading in Lake Hiidenvesi( southern Finland). Water Research, 2003, 37 : 4468-4474.

[14] 高镜清, 熊治廷, 张维吴等. 常见沉水植物对东湖重度富营养化水体磷的去除效果. 长江流域资源与环境, 2007,16 (6) :796-800.

[15] 刘臣炜,汪德爟. 湖泊富营养化内源污染的机理和控制技术研究. 农业环境科学学报,2006,25:814-818.

[16] 潘 纲,代立春,李 梁等. 改性当地土壤技术修复富营养化水体综合效果研究. 水质改善的应急与长期效果与机 制. 湖泊科学, 2012,24(6):801-810. 
[17] Coenye T, Nelis HJ. In vitro and in vivo model systems to study microbial biofilm formation. Journal of Microbiological Methods, 2010, 83: 89-105.

[18] Donlan RM. Biofilms: microbial life on surfaces. Emerging Infectious Diseases, 2002, 8: 881-890.

[19] Golladay SW, Sinsabaugh RL. Biofilm development on leaf and wood surfaces in a boreal river. Freshwater Biology, 1991, 25: $437-450$.

[20 ] Lorite GS, Rodrigues CM, de Souza AA et al. The role of conditioning film formation and surface chemical changes on Xylella fastidiosa adhesion and biofilm evolution. Journal of Colloid and Interface Science, 2011, 359 : 289-295.

[21] 国家环境保护总局《水和废水监测分析方法》编委会. 水和废水监测分析方法:第四版. 北京: 中国环境科学出版 社, $2002: 10$.

[22] 金相灿, 屠清瑛. 湖泊富营养化调查规范:第二版. 北京:中国环境科学出版社, 1990 .

[23] 徐骏. 杭州西湖底泥磷分级分布. 湖泊科学,2001, 13(3):247-254.

[24] Nguyen LM. Phosphate incorporation and transformation in surface sediments of a sewage-impacted wetland as influenced by sediment sites, sediment $\mathrm{pH}$ and added phosphate. Ecological Engineering, 2000, 14: 139-155.

[25] Roeselers G, van Loosdrecht MCM, Muyzer G. Phototrophic biofilms and their potential applications. Journal of Applied Phycology, 2008, 20: 227-235.

[26] McCormick PV, Shuford III RBE, Chimney MJ. Periphyton as a potential phosphorus sink in the Everglades Nutrient Removal Project. Ecological Engineering, 2006, $27: 279-289$.

[27] Chiou RJ, Yang YR. An evaluation of the phosphorus storage capacity of an anaerobic/aerobic sequential batch biofilm reactor. Bioresource Technology, 2008, 99: 4408-4413.

[28 ] Sainto LJ, Reddy KR. Biotic and abiotic uptake of phosphorus by periphyton in a subtropical freshwater wetland. Aquatic Botany, 2003, $77: 203-221$.

[29] Gottlieb A, Richards J, Gaiser E. Effects of desiccation duration on the community structure and nutrient retention of short and long-hydroperiod Everglades periphyton mats. Aquatic Botany, 2005, 82 :99-102.

[30 ] Wolf G, Picioreanu C, van Loosdrecht MCM. Kinetic model of phototrophic biofilms-the PHOBIA model. Biotechnology Bioengineering, 2007, 97 : 1064-1079.

[31] Varjo E, Liikanen A, Salonen VP et al. A new gypsum-based technique toreduce methane and phosphorus release from sediments of eutrophiedlakes: Gypsum treatment to reduce internal loading. Water Research, 2003, 37: 1-10.

[32] Wu Y, Zhang S, Zhao H et al. Environmentally benign periphyton bioreactors for controllingcyanobacterial growth. Bioresource Technology, 2010, 101: 9681-9687.

[33] 马天芳, 孙 青, 凌 媛等. 生物膜作用下沉积物一水界面溶液中 $\mathrm{pH}$ 和磷含量变化. 岩矿测试, 2011, 30 (6) : 683-687.

[34] Zhou AM, Wang DS, Tang HX. Phosphorus fractionation and bioavailability in Taihu Lake( China) sediments. Journal of Environmental Science, 2005, 17 (3) : 384-388.

[35] Edlund G, Carman R. Distribution and diagenesis of organic and inorgallic phosphorus in sediment of the Baltic proper. Chemosphere, 2001, 45: 1053-1061.

[36] 王春玲, 赵兴敏,赵兰坡. 富营养化水体内源磷释放和稳定化研究进展. 环境科学与管理, 2011,6(1):26-30.

[37] Hayashi M, Vogt T, Mächler L et al. Diurnal fluctuations of electrical conductivity in a pre-alpine river: Effects of photosynthesis and groundwater exchange. Journal of Hydrology, 2012, 450/451 : 93-104.

[38] 戴纪翠,宋金明,李学刚等. 胶州湾不同形态磷的沉积记录及生物可利用性研究. 环境科学, 2007,28(5):929-936.

[39］许金树,李亮歌. 台湾海峡中、北部沉积物中磷的存在形态. 海洋与湖沼, 1999,21 (1):62-69.

[40] Ting DS, Appan A. General characteristics and fractions of phosphorus in aquatic sediments of two tropical reservoirs. Water Science Technology, 1996, 34(7/8) : 53-59. 